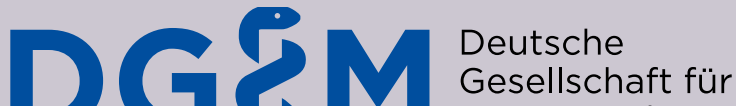 Innere Medizin
}

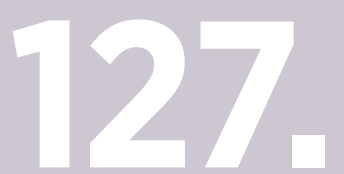

\section{Kongress der Deutschen Gesellschaft für Innere Medizin e.V.}

\author{
17. - 20. April 2021, Digital-Kongress
}

Von der Krise lernen

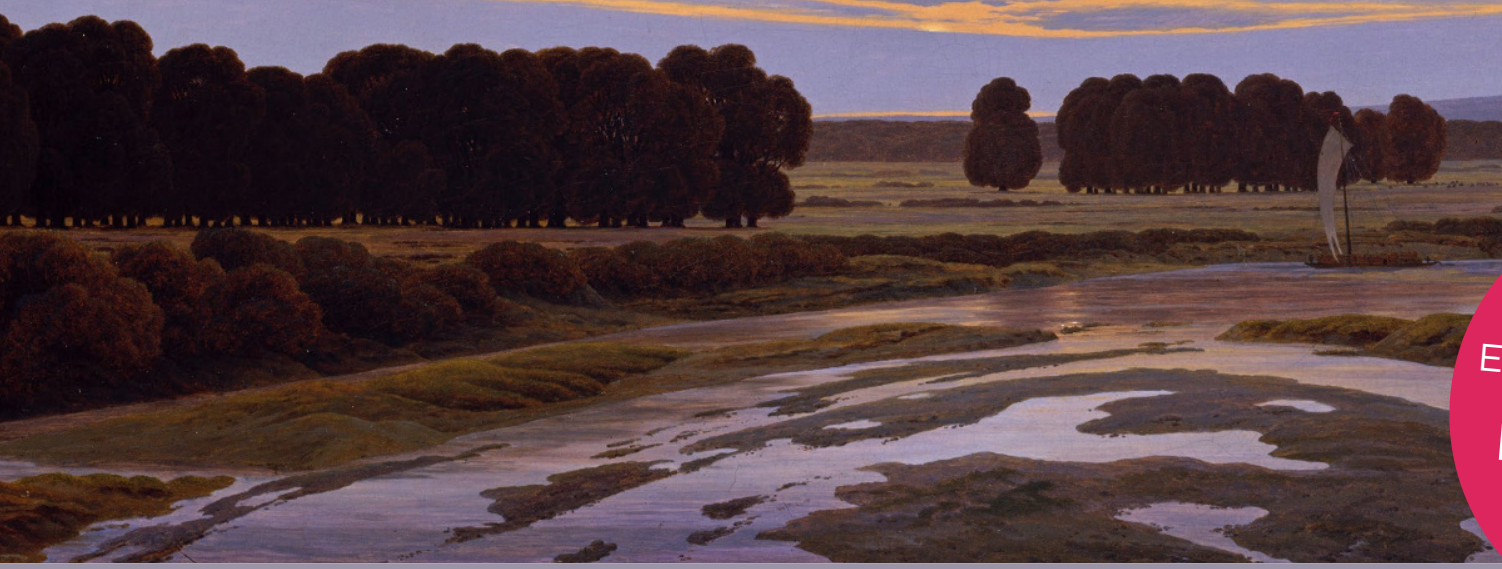

Vorsitzender der DGIM 2020/2021 und Präsident des 127. Kongresses

Prof. Dr. med. Sebastian Schellong

2. Medizinische Klinik

Städtisches Klinikum Dresden

DGIM - Kongress-Team 2021

Dr. med. Christian Stelzner (Kongresssekretär),

Dr. med. Cornelia Dostal,

Dr. med. Christoph Spranger,

Dresden

Prof. Dr. med. Viola Hach-Wunderle

Frankfurt/Main

Prof. Dr. med. Andreas Creutzig

Hannover
Kongress-Sekretariat

Ulrike Gerk

Silvia Hirt

dgim2021@klinikum-dresden.de

Leitthemen:

- Weniger ist mehr

- Gefäße verbinden

- Geschlechterunterschiede in der

Inneren Medizin

- Schnittstelle Psychosomatik

- Wandel der Behandlungsziele

-Wie lernt man Innere Medizin?

www.kongress.dgim.de
Hauptsponsor

Springer Medizin

Veranstalter \& Kongressagentur

$\mathbf{m}:$ con DIGITAL $-\frac{\pi}{\pi} \pi$
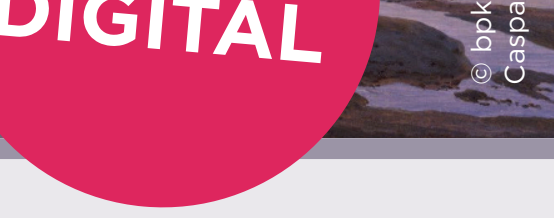


\section{Mitteilungen der DGIM}

Internist 2021 · 62:302-314

https://doi.org/10.1007/s00108-021-00977-3

๑) Springer Medizin Verlag GmbH, ein Teil von Springer Nature 2021

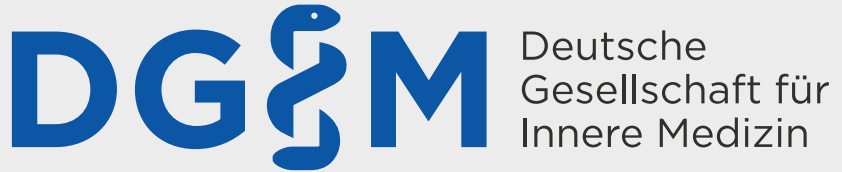

\section{Redaktion}

Prof. Dr. Georg Ertl (v.i.S.d.P.)

\section{Korrespondenzadresse}

Deutsche Gesellschaft für Innere Medizin e.V. (DGIM)

Irenenstraße 1

65189 Wiesbaden

Tel. 0611/205 8040-0 Fax 0611/205 8040-46

info@dgim.de

\begin{tabular}{|c|c|}
\hline 303 & $\begin{array}{l}\text { Klimawandel und Gesundheit - welche Verantwortung haben } \\
\text { Ärztinnen und Ärzte? Symposium auf dem DGIM Kongress } 2021\end{array}$ \\
\hline 304 & $\begin{array}{l}\text { DGIM: Patienten mit internistischen Vorerkrankungen früher gegen } \\
\text { COVID-19 impfen }\end{array}$ \\
\hline 306 & $\begin{array}{l}\text { Praxis-Ampel, Tipps zu Klimaschutz und Beratung für Kolleginnen } \\
\text { und Kollegen }\end{array}$ \\
\hline 306 & $\begin{array}{l}\text { Hausärztliche Internisten beim Kongress - DGIM und DEGAM tagen } \\
\text { gemeinsam }\end{array}$ \\
\hline 307 & "Viele waren überglücklich" \\
\hline 308 & 127. Internistenkongress \\
\hline 309 & $\begin{array}{l}\text { Das Forum Junge Internisten } 2021 \text { digital - mit vielen frischen } \\
\text { Themen }\end{array}$ \\
\hline 309 & Bündnis Junge Ärzte fordern mehr Nachhaltigkeit und Klimaschutz \\
\hline 310 & Nachruf Professor Dr. med. Karl Martin Koch \\
\hline 310 & Laborbefund nicht gesehen? Grober Organisationsfehler! \\
\hline 313 & Veranstaltungen unter der Schirmherrschaft der DGIM \\
\hline 314 & Intensivkurse Innere Medizin \\
\hline
\end{tabular}

\section{Klimawandel und Gesundheit - welche Verantwortung haben Ärztinnen und Ärzte? Symposium auf dem DGIM Kongress 2021}

Der Internistenkongress, Deutschlands größter Fachkongress, findet vom 17. bis 20. April 2021 erstmals rein digital statt. Aus aktuellem Anlass wird der DGIM-Kongress 2021 unter dem Leitthema:„,Von der Krise lernen" stehen. Dennoch knüpft er auch an Schwerpunktthemen des ausgefallenen Kongresses
2020 an. Dazu gehört auch eine Sondersitzung zu Klimawandel und Gesundheit.

Mit dem Klimawandel gehen unter anderem Temperatursteigerungen und Hitzewellen einher. Diese können sich etwa auf Blutdruck, Flüssigkeitshaushalt oder auch auf die Anfälligkeit für Wundinfektionen negativ auswirken. In einer hochkarätig besetzten Sonderveranstaltung unter Vorsitz von Dr. Eckart von Hirschhausen diskutieren Experten am Montag, dem 19. April 2021 zum Thema „Klimawandel und Gesundheit - welche Verantwortung haben Ärztinnen und Ärzte?". Zum Auftakt soll der wissenschaftliche Konsens zu den Entwicklungen des Klimawandels und seinen Auswirkungen auf die Gesundheit in Deutschland geklärt werden. Anschließend erörtern Professor Jürgen Floege, Kongress-Präsident der DGIM 2020, Dr. Klaus Reinhardt, Präsident der Bundesärztekammer, Sylvia Hartmann, Bund der Medizinstudierenden Deutschlands, Medizinethikerin PD Dr. Verina Wild und Professor Lothar Wieler, Präsident des Robert Koch-Instituts, Fragen wie: Welche gesellschaftliche Bedeutung hat das Thema, besonders für die Ärzteschaft/die Gesundheitsberufe?

\section{Terminhinweis}

Sondersitzung: Klimawandel und Gesundheit - welche Verantwortung haben Ärztinnen und Ärzte?

Datum: Montag, 19. April 2021

Uhrzeit: ab $10.45 \mathrm{Uhr}$

\section{Hauptthemen des 127. Internistenkongresses}

Unter dem Leitthema: „Von der Krise lernen“ und dem Schwerpunkt „Ärzte als Forscher" umfassen die Hauptthemen des 127. Internistenkongresses die folgenden Säulen:

1. Weniger ist mehr - Welche Leistungen sind verzichtbar und welche nicht, um tatsächliche Bedarfe zu decken und die vorhandenen Ressourcen - auch kurzfristig - an die richtige Stelle zu bringen? 2. Gefäße verbinden - Die Gefäßmedizin ist unverzichtbarer Partner von Gefäßchirurgie und Radiologie. Innerhalb der Inneren Medizin ist sie möglicher Bezugspunkt für pathophysiologische Konzepte, diagnostische Modalitäten oder therapeutische Ansätze.

3. Geschlechterunterschiede in der Inneren Medizin - Pathophysiologie, Exposition und geschlechtsabhängige medizinische Versorgung. 4. Schnittstelle Psychosomatik - In vielen Fällen ist eine Erkrankung oder Symptomkonstellation wesentlich von psychischen Faktoren bestimmt.

5. Wandel der Behandlungsziele - Die Technik der Therapiezieldefinition etabliert sich zu einem zentralen Handlungsfeld.

6. Wie lernt man Innere Medizin? - Wodurch zeichnet sich eine gute Ärztin oder ein guter Arzt aus? Und wie wird man es? Mehr Informationen sind unter www.kongress.dgim.de abrufbar 


\section{Stellungnahme zu STIKO-Empfehlungen}

\section{DGIM: Patienten}

\section{mit internistischen}

\section{Vorerkrankungen früher gegen COVID-19 impfen}

Nicht nur Menschen ab 80 Jahren, sondern auch jüngere mit bestimmten gravierenden Vorerkrankungen haben ein erhöhtes Risiko, schwer an COVID-19 zu erkranken und im schlimmsten Fall daran zu sterben. Um das Erkrankungs- und Sterberisiko in dieser Personengruppe zu senken, sollten sie daher frühzeitig eine COVID-19-Impfung erhalten. Dies fordert die DGIM gemeinsam mit den Schwerpunktgesellschaften der Inneren Medizin* und der Arbeitsgemeinschaft Wissenschaftlicher Medizinischer Fachgesellschaften (AWMF). Welche internistischen Krankheitsbilder mit einem erhöhten COVID-19-Risiko verbunden sind, haben Experten in einer aktuellen Stellungnahme zu den Impf-Empfehlungen der Ständigen Impfkommission (STIKO) zusammengefasst.

Da derzeit noch nicht genügend Impfstoff für alle Bürger zur Verfügung steht, hat die STIKO des Robert-Koch-Instituts (RKI) Empfehlungen ausgearbeitet, welche Personengruppen aufgrund eines besonders hohen Risikos für eine Ansteckung und einen schweren Krankheitsverlauf bevorzugt geimpft werden. Derzeit erhalten zunächst Personen über 80 Jahre, Bewohnerinnen und Bewohner in Alten- und Pflegeheimen sowie medizinisches Personal und Personal in der Altenpflege die Impfung. Vergangene Woche hat die STIKO eine Aktualisierung der Empfehlungen veröffentlicht. „Die aktualisierten Empfehlungen ermöglichen es Ärztinnen und Ärzten, Menschen nicht nur anhand des Alters, sondern auch anhand ihrer Vorerkrankungen einer der Priorisierungskategorien zuzuordnen“, sagt Professor
Dr. med. Georg Ertl, Generalsekretär der DGIM. „Wir begrüßen diese Aktualisierung ausdrücklich, denn bestimmte Vorerkrankungen erhöhen das Risiko für einen schweren Verlauf bei COVID-19 zum Teil deutlich", so der Würzburger Internist und Kardiologe.

$\mathrm{Zu}$ den besonders gefährdeten Gruppen zählen etwa Menschen mit gewissen Herz-Kreislauf-Erkrankungen, zum Beispiel mit einer koronaren Herzkrankheit, wenn sie durch die üblichen Maßnahmen nicht zu behandeln sind oder mit Herzinsuffizienz, aber auch Patienten mit Krebs oder Nierenpatienten an der Dialyse. „In internationalen Studien wurden bei diesen Patienten vergleichsweise viele schwere COVID-19-Verläufe und eine hohe Sterblichkeit re-

\section{Beteiligte Gesellschaften}

Deutsche Gesellschaft für Innere Medizin e.V.

Deutsche Gesellschaft für Geriatrie e.V.

Deutsche Gesellschaft für Infektiologie e.V.

Deutsche Gesellschaft für Internistische Intensiv- und Notfallmedizin e.V.

Deutsche Gesellschaft für Kardiologie - Herz- und Kreislaufforschung e.V.

Deutsche Gesellschaft für Hämatologie und Medizinische Onkologie e.V.

Deutsche Gesellschaft für Nephrologie e.V.

Deutsche Gesellschaft für Pneumologie e.V.

Deutsche Gesellschaft für Rheumatologie e.V.

Deutsche Gesellschaft für Gastroenterologie, Verdauungs- und Stoffwechselkrankheiten e.V.

Arbeitsgemeinschaft Wissenschaftlicher Medizinischer Fachgesellschaften (AWMF) gistriert", so Ertl. Wie das Risiko für einen schweren Krankheitsverlauf und eine erhöhte Sterblichkeit bei weiteren internistischen Vorerkrankungen ausgeprägt ist, hat die DGIM in der „Stellungnahme zur STIKOCOVID-19-Impfempfehlung einschließlich der aktuellen wissenschaftlichen Begründungen" unter Beteiligung der internistischen Schwerpunktgesellschaften ${ }^{1}$ aufgeführt. Dazu haben die beteiligten Experten die aktuelle Studienlage eingehend analysiert und ausgewertet.

Auf dieser Basis sprechen sich die DGIM und die beteiligten Gesellschaften dafür aus, Menschen mit Vorerkrankungen je nach Erkrankungsrisiko bevorzugt zu impfen - auch um Folgeschäden zu vermeiden. „Denn gerade Menschen mit chronischen Erkrankungen schotten sich aus Angst vor einer Infektion sozial ab oder nehmen auch unbedingt notwendige medizinische Untersuchungen nicht wahr", erläutert Professor Dr. med. Sebastian Schellong, Vorsitzender der DGIM und Chefarzt der zweiten Medizinischen Klinik am Städtischen Klinikum Dresden. Ein früherer Zugang zur Impfung könnte dabei helfen, bei diesen Patienten das Risiko einer COVID-19-Erkrankung sowie von Folgeschäden durch eine ausbleibende medizinische Versorgung zu senken, so Schellong.

Zur vollständigen Stellungnahme gelangen Sie hier: https:// www.dgim.de/fileadmin/user upload/PDF/Publikationen/Archiv/Positionspapiere_und_Stellungnahmen/FINAL_DGIM_ 20210107_Stellungnahme_ STIKO-COVID-19.pdf
Ordentliche Mitgliederversammlung der Deutschen Gesellschaft für Innere Medizin e.V.

Sonntag, 18. April 2021, 14.30-16.00 Uhr

Kurhaus Wiesbaden, Kurhausplatz 1, 65189 Wiesbaden

Tagesordnung

1. Bericht des Vorsitzenden

2. Bericht des Generalsekretärs

3. Bericht des Geschäftsführers

4. Kassenbericht

5. Entlastung des Vorstandes, des Kassenführers und des Geschäftsführers

6. Wahlen zum Vorstand

7. Wahlen zum Ausschuss

8. Wahlen von Ehrenmitgliedern

9. Wahlen der Mitglieder für die Vorschlagskommission zur Wahl von Vorstand und Ausschuss

10. Verschiedenes

11. Amtsübergabe

Prof. Dr. med. Sebastian Schellong

Vorsitzender 2020/2021 


\section{Medizinstudierende Deutsche Gesellschaft für Innere Medizin e.V.}

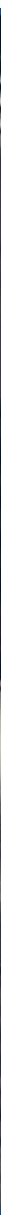

Beantrage als Medizinstudent/in ab dem 6. Fachsemester Deinen kostenfreien DGIM-Gastzugang für die Deutsche Gesellschaft für Innere Medizin e.V., die mit über 27.000 Mitgliedern zu den größten medizinisch-wissenschaftlichen Fachgesellschaften in Europa zählt, und nutze die zahlreichen Vorteile:

> Freier Eintritt zum jährlichen Internistenkongress (auch digital)

> Einreichung von Abstracts für die jährliche Preisverleihung auf dem Internistenkongress

> Erhalt des Kongress-Newsletters sowie E-MailInformationen der Gesellschaft

> Kostenloser Bezug der Zeitschrift Der Internist (via Onlinezugriff oder Printausgabe)

> Onlinefortbildung in der DGIM e.Akademie unter www.dgim-eakademie.de
> Teilnahme an der Ausschreibung der

\section{Promotionsstipendien}

> Möglichkeit der Mitarbeit in der Arbeitsgruppe der Jungen Internisten der DGIM

> Erhalt des Aspire-Newsletters mit Themen für junge Internisten

> Beratung zu Weiterbildungsthemen und berufsrelevanten Rechtsfragen

> Nutzung der DGIM-Vorteilsangebote 


\section{Praxis-Ampel, Tipps zu Klimaschutz und Beratung für Kolleginnen und Kollegen}

\section{Angebote der AG Hausärztliche Internisten}

Die AG Hausärztliche Internisten hat vor Kurzem einige Neuerungen auf den Weg gebracht. So hat die Arbeitsgruppe Empfehlungen zur individuellen Umstrukturierung des Praxismanagements in der CoronaPandemie ausgearbeitet und auf der DGIM-Webseite veröffentlicht.

Die zentralen Bausteine des Influenzapandemie-Risikomanagements in Arztpraxen (Empfehlung der KBV, BÄK und der BGW von 2008) umfassen neben Hygieneregeln insbesondere die Punkte „Patientenströme trennen“ und „Reduzierung der Konsultationen in der Praxis" heißt es in den Empfehlungen. In der aktuellen Pandemie gäbe es wechselnde Risiko-Szenarien abhängig von den Neuinfektionszahlen im zeitlichen und örtlichen Verlauf. Hierfür soll deshalb eine Praxis-Ampel Orientierung bieten, um die Praxisorganisation individuell den wechselnden Risikosituationen und den Praxisbedingungen anpassen zu können (siehe dazu Infokasten).

Zudem hat die AG Tipps für eine nachhaltige Praxis und eine Liste mit Anregungen zum Thema Klimaschutz und Gesundheit zusammengestellt. Darin wird aufgeführt, wie Praxisärzt* innen zum Beispiel Energie sparen, mit Büromaterial und Verbrauchsmaterialien schonender umgehen und die Mobilität überdenken können. Genaue Informationen dazu gibt es unter www.dgim.de/ueber-uns/hausaerztliche-internisten/

Mitglieder der Arbeitsgemeinschaft bieten zudem eine kollegiale Beratung für Ärztinnen und Ärzte, die sich ggf. niederlassen wollen und hierzu viele Fragen haben. Weitere Informationen dazu gibt es unter www.dgim.de/ueber-uns/hausaerztliche-internisten/kollegiale-beratung/

\section{Hausärztliche Internisten beim Kongress - DGIM und DEGAM tagen gemeinsam}

Vor dem Hintergrund des demografischen Wandels, einer komplexer werdenden Medizin und schwindenden personellen Ressourcen kommen auf die Hausärzte in den nächsten Jahren gewaltige Aufgaben zu. Um diese anzugehen, ist eine gute $\mathrm{Zu}$ sammenarbeit auch der Fachgesellschaften untereinander von großer Bedeutung. Um diese Entwicklung zu unterstützen, finden gemeinsame Symposien der AG Hausärztliche Internisten mit der Deutschen Gesellschaft für Allgemeinmedizin und Familienmedizin e. V. (DEGAM) statt.

Patienten mit rheumatischen
Erkrankungen - wer darf
sie behandeln und wenn ja,
wie? Ein interdisziplinärer
Workshop zur Schnittstelle
Hausarzt/Facharzt
Datum: Sonntag, der 18. Ap-
ril 2020
Uhrzeit: 14.45 Uhr
Vorsitz: Dr. Till Uebel (Neckar-
gemünd) und Dr. Christine
Schwill (Bad Schwartau)

\section{Terminhinweise (Auswahl)}

Schnittstelle Hausarzt und niedergelassener Kardiologe (gemeinsame Sitzung von DGIM und DEGAM)

Datum: Sonntag, der 18. April 2021

Uhrzeit: ab $13.00 \mathrm{Uhr}$

Vorsitz: Dr. med. Eva Hintz (frühere Vorsitzende der AG Hausärztliche Internisten der DGIM, Praxis für Innere Medizin, Winnenden)

Professor Dr. med. Erika Baum (DEGAM, Philipps-Universität Marburg)

\section{Patienten mit rheumatischen}

\section{Die Corona Praxis Ampel \\ GRÜN $=<50$ Neuinfektionen/100.000 Einwohner \\ - separate Infektionssprechstunde (räumlich/zeitlich getrennt) \\ - im Wesentlichen nur Bestellsprechstunde organisieren und entspre- chend kommunizieren \\ - ansonsten Normalbetrieb mit Einhaltung der AHA Regeln (MNS-Masken für Patienten und Personal, je nach Tätigkeit auch FFP2-Maske) \\ - normales bearbeiten von Routineterminen, einschließlich DMP \\ - akut Patienten noch großzügig zulassen, wenn Patientenzahl in Praxis kontrollierbar \\ GELB $=>50$ Neuinfektionen/100.000 Einwohner \\ - separate Infektionssprechstunde (räumlich/zeitlich getrennt) \\ - alle Praxiskontakte nur noch mit Termin \\ - Video- und Telefontermine anbieten, telefonische AU bei leichten resp. Infekten \\ - Dauerrezepte möglichst nur noch postalisch \\ - Patienten auffordern Routinetermine zu verschieben}

- Akut Patienten nur bei akuten Schmerzen/Kreislaufstörungen in separatem Raum

- Patientenanzahl im Wartezimmer auf max. 4 Patienten, bzw. je nach Abstandsmöglichkeit reduzieren

- Arbeiten mit FFP2-Masken dauerhaft

ROT > 200 Neuinfektionen/100.000 Einwohner bzw. unkontrollierte Pandemielage

- Infektionssprechstunde dem Bedarf zeitlich anpassen und erweitern

- Routinetermine bei stabilen Patienten werden aktiv durch Praxis abgesagt

- Ausweitung der Videosprechstunde

- dauerhaftes arbeiten mit FFP2-Maske

- Freistellung von Praxispersonal aus Risikogruppen für patientennahe Arbeiten

- ggf. $24 \mathrm{~h}$-Notfall-Praxis-Handy mit Beratung durch Praxisarzt

- ggf. Aufteilung des Praxispersonals auf Team A und B

- ggf. Erweiterung der Sprechzeiten auf Samstag 


\section{„Viele waren überglücklich“}

\author{
Hausärztin Dr. Irmgard Landgraf
}
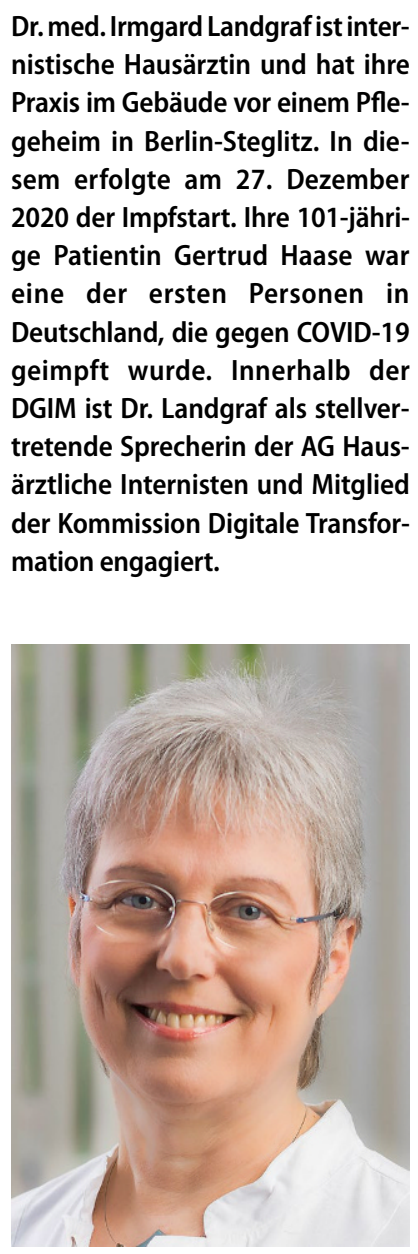

Sehr geehrte Frau Dr. Landgraf, aus Ihrer täglichen Arbeit als Hausärztin im Sophienhaus und weiteren Pflegeheimen haben Sie einen sehr direkten Einblick in die Auswirkungen der Pandemie. Wie haben Sie die Corona-Pandemie bislang erlebt?

COVID-19 ist eine Infektion, die uns alle bedroht und vor allem dadurch gefährlich ist, dass insbesondere ältere Menschen ein hohes Risiko haben, daran schwer zu erkranken oder zu versterben.

Um unsere alten und multimorbiden Pflegeheimbewohner zu schützen, habe wir vieles unternommen. Dazu gehörte, AuBenkontakte und Besuche zu reduzieren. Obwohl unsere Pflegekräfte alles unternommen ha- ben, um die Heimbewohner für die nicht möglichen Besuche zu entschädigen, viele Beschäftigungsangebote gemacht haben, die Angehörigen über Videotelefonie „ins Heim geholt“ und den Kontakt über digitale Möglichkeiten unterstützt haben, war es für alle eine harte Zeit.

Besonders in Alten- und Pflegeheimen gab es in den letzten Monaten immer wieder COVIDAusbrüche mit hohen Todeszahlen. Das damit verbundene Leid ist auch deshalb groß, weil die betroffenen Menschen unter Quarantänebedingungen sterben mussten, ohne Begleitung ihrer Angehörigen.

Deshalb finde ich die vom RKI umgesetzte Strategie, mit dem noch nicht für alle Menschen vorhandenen Impfstoff zunächst Pflegeheimbewohner und Menschen über 80 Jahre zu impfen, genau richtig. Denn so können wir viele Todesfälle verhindern.

Wie liefen die Vorbereitungen auf den Impfstart am 27. Dezember 2020 im Sophienhaus und ist bei der Impfung selbst alles gut verlaufen?

Nur eine Woche vor der geplanten Impfung wurde uns der genaue Impftermin bestätigt. Wir standen sehr kurzfristig vor der Aufgabe, alle Heimbewohner beziehungsweise ihre Angehörigen über die Impfung aufzuklären und ihre Einwilligung dazu einzuholen. Da etwa $80 \%$ unserer Pflegeheimbewohner an einer mehr oder weniger ausgeprägten Demenz leiden, müssen Angehörige oder Betreuer Entscheidungen $\mathrm{zu}$ ihrer gesundheitlichen Versorgung treffen. Daher haben Heimleitung, Pflegekräfte und Servicemitarbeiter diese über die geplante Impfung informiert und ihnen die notwendigen Formulare ausgehändigt.

Als Hausärztin stand ich den Angehörigen und Betreuern für Fragen zur Verfügung.
Da der Beratungs- und Aufklärungsbedarf groß war, habe ich während der Weihnachtsfeiertage viele Telefonate geführt.

Die Impfung selbst verlief unkompliziert und ohne Probleme. Insgesamt haben alle die Impfung sehr gut vertragen. Auch in den Folgetagen sind keine unerwünschten Impfreaktionen aufgetreten.

Wie haben die Bewohner des Pflegeheims auf die Möglichkeit reagiert, die Impfung als erste $z u$ bekommen?

Die Bewohner, die die Bedeutung der Impfung erfasst haben, waren sehr froh, zu den Ersten zu gehören, die dadurch vor einem schweren COVID-19-Verlauf geschützt werden. Die vielen Angehörigen, die ich vorab beraten haben, waren meist überglücklich. Es war, als würde ein über uns schwebendes Damoklesschwert weggenommen. Wir haben ja in den letzten Monaten vieles unternehmen müssen, um unsere Heimbewohner vor einer Corona-Infektion zu schützen und hatten trotzdem immer Sorgen, dass SARS-CoV-2 doch einmal eingeschleppt wird.

Sie betonen immer wieder den großen Nutzen digitaler Pflegeakten und der Telemedizin in der aktuellen Situation. Worin bestehen die konkreten Vorteile?

Digital vernetzt zusammenzuarbeiten bedeutet, dass wir Pflegekräfte und Ärztinnen die digitalen Akten für unsere Kommunikation und den wichtigen Informationsaustausch sektorenübergreifend nutzen, wann immer das möglich ist. Dadurch sparen wir Zeit, die wir gerade jetzt während der Pandemie mit dem deutlich größeren Beratungsbedarf und den intensiveren Hygienemaßnahmen bei gleichzeitigem Personalmangel gut gebrauchen können.

Außerdem haben wir Ärztinnen während der Pandemie viel mehr digitale Kurvenvisiten anstelle von Visiten vor Ort durchgeführt und auch damit das Infektionsrisiko für die Pflegeheimbewohner reduziert. Unsere Patienten waren trotzdem genauso engmaschig betreut wie vorher. Auch digital können wir rasch auf Beschwerden durch diagnostische oder therapeutische Maßnahmen reagieren und dafür sorgen, dass es den Bewohnern gut geht. In anderen Heimen, wo nicht selten 20 bis 30 Ärzte für die Bewohner zuständig sind, ist das natürlich nicht so einfach.

Als Hausärztin besuchen Sie normalerweise auch viele ältere $\mathrm{Pa}$ tienten zu Hause. Was für Schwierigkeiten bestehen hierbei aktuell und wie lassen sich diese eventuell lösen?

Gerade bei älteren und multimorbiden Patienten, die zu Hause von Angehörigen und Pflegediensten versorgt werden müssen und das Haus nicht verlassen können, ist die COVID-19-Impfung mit der aktuellen Strategie nicht durchführbar. Sie sind zu krank, um ins Impfzentrum gefahren zu werden. Die Impfärzte der mobilen Impfteams machen keine Hausbesuche bei einzelnen $\mathrm{Pa}$ tienten. Hier hoffe ich, dass wir Hausärztinnen und Hausärzte unsere zu Hause gepflegten $\mathrm{Pa}$ tienten bald mit dem in Aussicht gestellten Impfstoff impfen können, der sich fünf Tage unter normalen Kühlschrankbedingungen und somit auch in Praxen aufbewahren lässt. Ich würde diese Impfung dann auch den älteren und chronisch kranken Patienten anbieten, die zwar noch mit Mühe $\mathrm{zu}$ mir in die Praxis kommen können, denen aber die Fahrt ins Impfzentrum nicht mehr zumutbar ist. Wichtig ist, dass vor allem die gefährdete Bevölkerungsgruppe möglichst umfangreich geimpft ist.

Eines weiß ich sicher: Sobald COVID-Impfungen in Hausarztpraxen durchführbar sind, wird die Impfquote noch einmal deutlich steigen - und zwar bei Menschen jeden Alters, vor allem aber bei den älteren Menschen, die den Impfschutz ja ganz besonders dringend benötigen. 


\section{Internistenkongress 17. bis 20. April 2021}

\section{Frühjahrssymposium der Korporativen Mitglieder}

Sonntag, 18. April 2021, 14.4517.00 Uhr (Online-Veranstaltung)

„Ärztliche Information und deren Dissemination im digitalen Wandel“

Vorsitz:

Dr. Daniel Kalanovic, Senior Medical Director Germany, Pfizer, Berlin

Dr. Paul Herrmann, Director Journals \& ePublishing, Springer Medizin, Heidelberg

\section{Vorläufige Themen und Referenten}

Einführung ins Thema (14.45$14.50 \mathrm{Uhr}$ )

Dr. Daniel Kalanovic, Senior Medical Director Germany, Pfizer, Berlin

Dr. Paul Herrmann, Director Journals \& ePublishing, Springer Medizin, Heidelberg

Entwicklungen von Wissenschaftspublikationen (14.5015.10 Uhr)

Dr. Max Voegler, Vice President Global Strategic Networks $\mathrm{DACH}$, Elsevier, Berlin
Herausforderungen beim wissenschaftlichen Publizieren (15.10-15.30 Uhr)

Daniel Tiemann, Vice President STM, De Gruyter, Berlin

Wandel der Rolle und Funktion von Fachzeitschriften (15.3015.50 Uhr)

Prof. Dr. Christopher Baethge, Leiter der Medizinisch-Wissenschaftlichen Redaktion des Deutschen Ärzteblatts, Köln

Was ärztlicher Content heute leisten muss/Wie sich das Nutzerverhalten auf die Gestaltung von Arztinformation auswirkt (15.50-16.10 Uhr)

Dr. Cécile Mack, Director Digital Products \& Marketing, Springer Medizin, Berlin
Wie verändern sich ärztliche Berufsbilder durch die digitale Transformation? Beispiele aus der Praxis (16.10-16.30 Uhr) Dr. Dierk Heimann, FA für Allgemeinmedizin und Medizinjournalist, Mainz

Diskussion (16.30-17.00 Uhr)

\section{Hygienefortbildung kosten- und zeiteffizient.}

\section{Zertifizierte Fortbildungen für Ärzte}

Online und mobil - Auf allen Endgeräten und ohne Softwareinstallation Smart-Learning $₫$ Kurse zum Erwerb anerkannter Qualifikationen

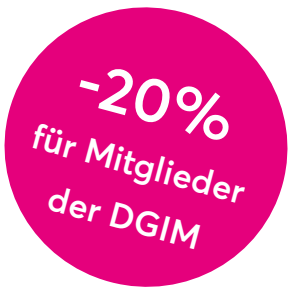

Hygienebeauftragter Arzt

Nach dem Curriculum der

Bundesärztekammer

60 CME-Punkte
Patientenaufklärung

Das Update für Qualität und

Sicherheit in Klinik und Praxis

2 CME-Punkte
Kommunikation im Schadenfall
Leitfaden für das klinische

Risikomanagement

2 CME-Punkte

\section{Jetzt buchen:}




\section{Das Forum Junge Internisten 2021 digital - mit vielen frischen Themen}

\begin{abstract}
Die Arbeitsgruppe Junge Internisten der DGIM vertritt den internistischen Nachwuchs inner- und außerhalb der Fachgesellschaft. Zielpublikum des „Forum Junge Internisten“ sind vor allem - aber bei Weitem nicht nur - Medizinstudierende und Junge Ärzte in der Weiterbildung. Neben einer praxisnahen Darstellung von Themen aus dem klinischen Alltag werden Aspekte der ärztlichen Weiterbildung, Möglichkeiten einer wissenschaftlichen Karriere und Themen wie Frauen in der Medizin und die Vereinbarkeit von Beruf und Familie diskutiert.
\end{abstract}

auch eine Session im Hauptprogramm aus. Die Referenten nähern sich aus verschiedenen Blickwinkeln der großen Frage unseres Fachs: „Wie wird man ein guter Internist?".

Die DGIM unterstützt wissenschaftliches Arbeiten und Forschen durch mehrere attraktive Stipendien. Wir freuen uns daher sehr, dass die Stipendiaten ihre Arbeiten im Rahmen des Forums vorstellen werden.

Die Corona-Pandemie hat der Digitalisierung im Gesundheits- wesen großen Schwung verliehen. Wir freuen uns sehr, dass der Kongress in diesem Jahr in digitaler Form stattfinden kann und sind vom Erfolg dieses Formates überzeugt. Schauen Sie gerne regelmäßig in unserem Programm vorbei und diskutieren Sie mit wir freuen uns auf Sie!

Dr. med. Matthias Raspe, Dr. med. Anahita Fathi und Dr. med. Moritz Hundertmark (Sprecher/-in der AG Junge Internisten)

\section{Das digitale Programm des Jungen Forums 2021}

Samstag, 17.04.2021

Meet the ...

Studientelegramm live

Der Internist im postfaktischen Zeitalter

Hätten Sie es gewusst? - Spannende Fälle aus den europäischen

Schools für Innere Medizin

Rollenmodelle - Starke Frauen in der Medizin

Podiumsdiskussion: „Führungskräfte in leitender Position: Medizin trifft Wirtschaft"

Ein neues Lehrbuch? Von der Idee zum erfolgreichen Standardwerk

Sonntag, 18.04.2021

Meet the ...

Wunsch und Wirklichkeit im Krankenhaus - Aufruhr in der neuen

Generation

Fälle aus der DGIM eAkademie

Innere Medizin interdisziplinär - Häufige Fragen von Internisten

Der Young Investigator Award

Best of DGIM - das Clinician Scientist Programm

Montag, 19.04.2021

Meet the ...

Wie up to date bleiben?

Wissenschaftliches Schreiben

Karrierewege in der Forschung - Von Höhen und Tiefen

Interessante Einblicke in die Arbeit des ABS-Teams

Best of DGIM - Spitzenforschung in der Inneren Medizin

Best Practice Weiterbildung: Kliniken stellen ihr Erfolgskonzept vor

Dienstag, 20.04.2021

Meet the ...

Innere Medizin und Social Media - Chance oder Risiko

Toolbox Angiologie \& Rheumatologie

Arbeiten in Teilzeit - geht das überhaupt?

Infektiologisches Quiz der Blickdiagnosen
In seinem neusten Positionspapier fordert das Bündnis Junge Ärzte (BJÄ) „unverzüglich und mit konkreten und sinnvollen Maßnahmen an der Umsetzung von Klimaschutzzielen“" im Gesundheitswesen mitzuwirken. Dort heißt es unter anderem, dass der „Gesundheitssektor [...] im Jahr 2017 für knapp $5 \%$ des weltweiten Ausstoßes klimaschädlicher Gase verantwortlich"war.

Trotz internationaler Vereinbarungen, wie etwa dem Pariser Klimaschutzabkommen, ist die dringend notwendige Emissionstrendwende weiterhin nicht abzusehen. „Extremwetterereignisse, Überschwemmungen, Dürren, Temperaturanomalien, Waldbrände, Biodiversitätsverlust, Infrastrukturschäden und "neue“ Infektionskrankheiten sind bereits konkrete Bedrohungen der Gegenwart", so die BJÄ-Autoren. Daher setzt sich das BJÄ für eine „klimagerechte Umgestaltung der Gesundheitssysteme ein “ und ruft zu „besonderem Engagement im Klimaschutz auf".

Das BJÄ ist als jüngere Generation aktuell und zukünftig betroffen und fordert, die „vom
Europäischen Parlament verschärften Klimaziele für 2030 $\mathrm{zu}$ verwirklichen, Nachhaltigkeit privat und beruflich umzusetzen und für die Sicherung globaler Gesundheit einzutreten". Weiter werden konkrete Maßnahmen vorgeschlagen, wie etwa die Übernahme einer Vorbildfunktion als Repräsentant des Gesundheitswesens, Stärkung der interdisziplinären und intersektoralen Zusammenarbeit für den Klima- und Gesundheitsschutz zur Nutzung von Synergieeffekten, Unterstützung konkreter Klimaschutzmaßnahmen in lokalen Gesundheitseinrichtungen oder Einforderung klimaneutraler Finanzanlagen und Investitionen z. B. durch die Ärzteversorgung.

Dafür, so die BJÄ-Autoren, „müssen die verantwortlichen Akteure und Gremien vorangehen und damit einen Einstellungs- und Lebenswandel in der Gesamtbevölkerung fordern und fördern".

Das ganze Positionspapier finden Sie hier: https://www.buendnisjungeaerzte.org/fileadmin/ user_upload/PDF/2021_01_Positionspapier_Klima_Gesundheit.pdf 


\section{Nachruf Professor Dr. med. Karl Martin Koch}

Am 22. Dezember 2020 ist im Alter von 86 Jahren Professor Dr. med. Karl Martin Koch verstorben. Mit ihm geht ein weiterer Pionier, der die Entwicklung der Nephrologie in Deutschland nachhaltig geprägt hat.

Karl Koch wurde 1934 in Eschwege in einen Arzthaushalt hinein geboren. Er studierte Medizin in Göttingen, Würzburg und Frankfurt. Dort kam er auch in der Medizinischen Klinik von J. Frey in Kontakt mit der Nierenphysiologie. Erste Arbeiten zusammen mit Klaus Hierholzer, der später den Lehrstuhl für Klinische Physiologie an der Freien Universität besetzen sollte, kreisten um die Pathophysiologie des Elektrolythaushalts. Heute kaum noch vorstellbar, hatten sie als modernstes Gerät ein Flammenphotometer zur Hand. Zum Erwerb einer soliden grundlagenwissenschaftlichen Ausbildung ging er 1964 von Frankfurt an das renommierte Max-Planck-Institut für Experimentelle Medizin in Göttingen in den Arbeitsbereich von Professor Bruno Ochwadt. 1966 wechselte er mit einem Stipendium an das New York University Hospital zu Professor Norman Bank, wo er seine Arbeiten zu Hypertonie und Natriurese fortsetzte und die Ergebnisse im J. Clin. Invest. publizierte.

Als er 1968 nach Frankfurt zurückkehrte, wurde er mit ei-

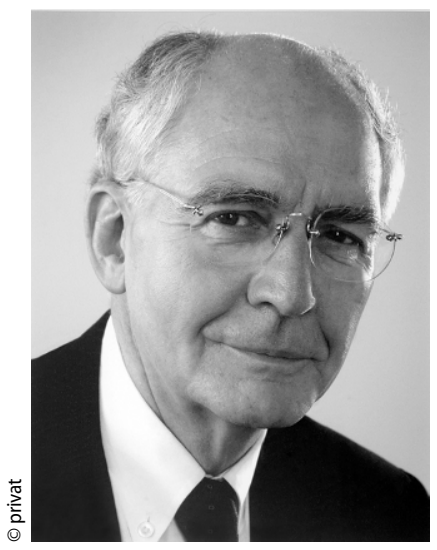

$\Delta$ Prof. Dr. med. Karl Martin Koch nem massiven klinischen Problem konfrontiert, der Therapie terminal niereninsuffizienter $\mathrm{Pa}$ tienten. Nur ein kleiner Teil von ihnen konnte behandelt werden, weil klinische Behandlungsplätze fehlten. In dieser Situation machte er die Bekanntschaft des Pioniers der Heimdialyse, Stanley Shaldon in London, der für den jungen, bislang nur in Pathophysiologie geschulten Karl Koch zum Mentor in der Urämiebehandlung wurde. Zusammen mit Wilhelm Schoeppe, Klaus Ketzler und anderen gründeten sie 1969 das Kuratorium für Heimdialyse. Mit diesem Verein konnte zunächst über die Heimdialyse und später in Zentren das Versorgungsproblem erfolgreich angegangen werden.

Doch dem geschulten Physiologen fielen mehrere Probleme des dialysierten Patienten auf. Und so wandte er sich auch der Pathogenese der renalen Anämie zu, ein Thema, das mit der Verfügbarkeit von Erythropietin ein vorläufiges Ende fand. Neben den Störungen des Calcium-Phosphatstoffwechsel interessierte er sich darüber hinaus für das bei der damaligen Dialyse oft auftretende Fieber, das bis dato als banale Nebenwirkung der Maschienenbehandlung gesehen wurde. In Zusammenarbeit mit Charles Dinarello in Boston entstand die „Interleukin-1 Hypothese", nach der im Dialysat enthaltenen bakteriellen Toxine in der Lage waren, durch die Dialysemembran hindurch systemische Entzündungsbotenstoffe zu induzieren. Damit wurde die Grundlage für das heute übliche, weitgehend sterile Dialysat gelegt.

Daneben setzte er seine akademische Karriere fort, 1971 habilitierte er sich für das Fach Innere Medizin und wurde im Folgejahr zum Professor ernannt. 1982 wurde er auf den renommierten Lehrstuhl für Nephrologie an der Medizinischen Hochschule Hannover (MHH), den vor ihm Professor Jan Brod innehatte, berufen. Dort ent- standen die entscheidenden Arbeiten zur chronischen Inflammation des Dialysepatienten. Daneben etablierte seine Arbeitsgruppe wichtige Beiträge der Nephrologie in der Nierentransplantation zusammen mit R. Pichlmayr, so zur chronischen Dysfunktion von Transplantaten und zur Indikationsausweitung auch im Alter.

Ab 1989 übernahm er auch Verantwortung in den Gremien der MHH, so war er von 1989 bis 1993 Ärztlicher Direktor der MHH und von 1997 bis zu seinem Ruhestand im Jahre 1999 Rektor.

Karl Koch hinterlässt ein großes wissenschaftliches Oeuvre mit einer weiten Spanne von der Mikropunktion bis zur PCR und eine große Zahl von Schülern, die an Universitäten und Krankenhäusern seine Haltung und $\mathrm{He}$ rangehensweise weiterverbreiteten. Kurz vor Weihnachten ist er nach längerer Krankheit verstorben. Seine Mitarbeiter verneigen sich respektvoll vor einem großen Kliniker und Forscher der deutschen Nephrologie.

Deutsche Gesellschaft für Innere Medizin e.V.

Prof. Jürgen Floege, Aachen Prof. Ulrich Frei, Berlin

\section{Laborbefund nicht gesehen? Grober Organisationsfehler!}

Nicht zur Kenntnis genommene Laborwerte führten adäquat-kausal bei einer Patientin (Jg. 1985) zum Tod durch Hirninfarkt. Durch Beschluss des OLG Koblenz vom 25.09.2017 (5 U 427/17) ist die Zurechnung der kausalen Schadensfolgen aus dem Behandlungsfehler auch auf nachbehandelnde Ärzte erweiterbar.

Die Kläger sind die Eltern der verstorbenen Patientin. Mit Blick auf eine anstehende Kieferoperation wurde bei der Patientin eine Blutentnahme zur Untersuchung der Laborwerte vorgenommen. Die Laborwerte zeigten eine Anämie und eine Leukopenie und das Differenzialblutbild eine Anisozytose, auffällige Riesenthrombozyten und drei Blasten sowie eine Poikilozytose. Nur hatte der betreuende Hausarzt diesen Befund nicht zur Kenntnis genommen. Obwohl die Patientin zur Besprechung der Blutwerte in der Sprechstunde erscheinen sollte, wurden der Patientin auf ihr nachdrückliches Verlangen die Werte einfach ohne Besprechung mit dem Arzt ausgehändigt.

Nach Ansicht des OLG Koblenz liegt ein grober Organisa- tionsfehler vor, wenn nicht sichergestellt wird, dass bei der hausärztlichen Versorgung ein Laborbefund sowie die in der Praxis erhobene Blutsenkungsgeschwindigkeit auch ohne $\mathrm{Pa}$ tientenkontakt zur Kenntnis genommen, ausgewertet und erforderlichenfalls nach Kontaktaufnahme zum Patienten mit diesem besprochen werden. Dass hier die Patientin den Laborbefund einfach abholt und ein Gespräch verhindert habe, begründet alleine kein Mitverschulden der Patientin, sondern geht voll zu Lasten des Arztes.

Der nachbehandelnde Arzt lenkte das durch die Fehlbehandlung des Hausarztes entstandene Risiko auch nicht in eine völlig neue Richtung. Damit stand sein Fehlverhalten gleichwertig neben dem des erstbehandelnden Arztes.

Einmal mehr zeigt sich, wie wichtig die Kenntnisnahme jeglicher Laborbefunde und die dazugehörige Information des $\mathrm{Pa}$ tienten und Dokumentation des Patientenkontaktes ist.

Maximilian Gehrmann Rechtsrefendar 


\section{www.DGIM-eAkademie.de}

Das Fortbildungs-Portal der DGIM: Kostenfrei für alle Mitglieder

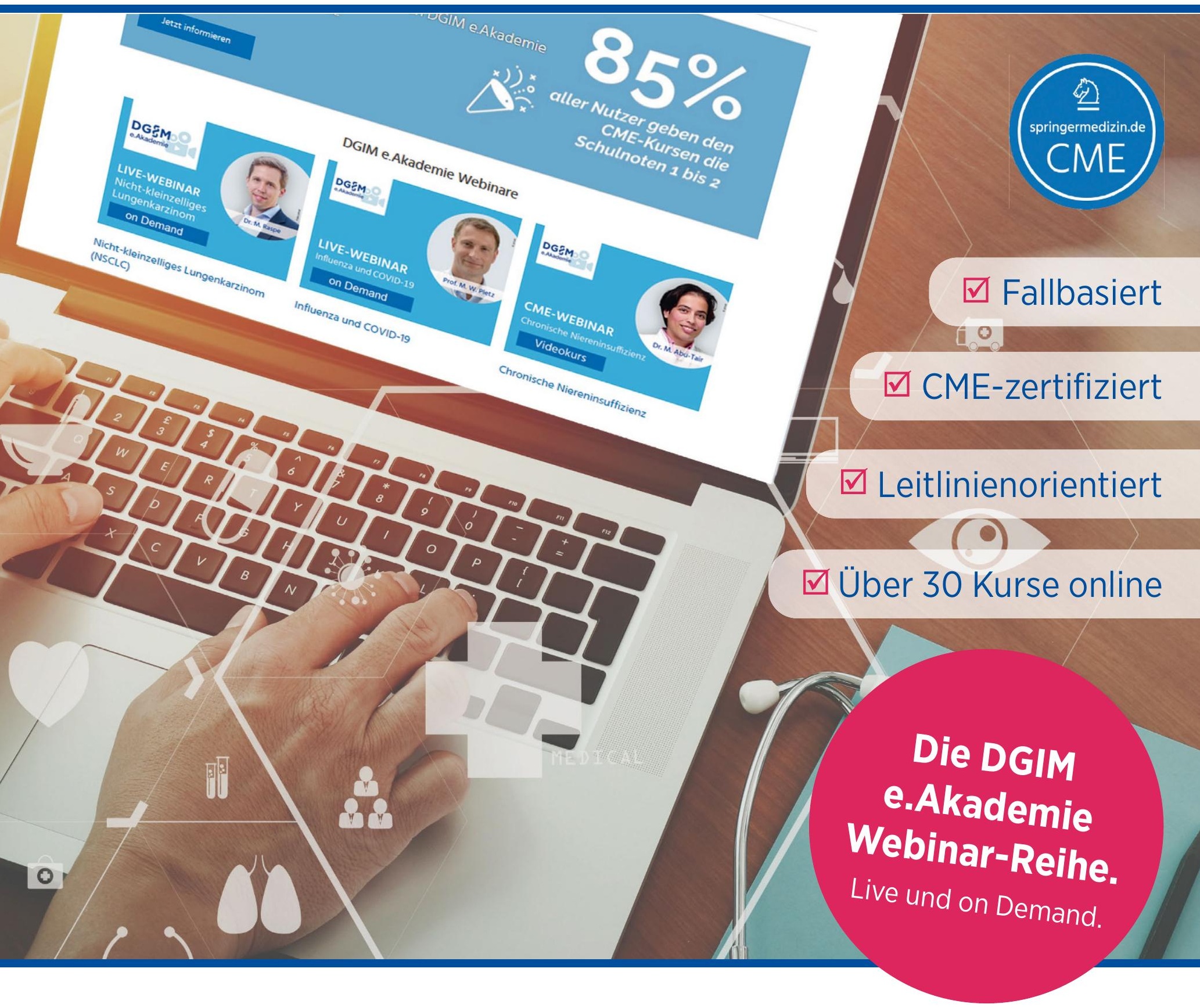

Entdecken Sie die Webinare* und interaktiven Kurse für Internisten zu aktuellen Leitlinien

*Webinare werden im Nachgang zertifiziert 


\section{INFEKTIO UPDATE 2021}

\section{Aktuell, kompakt und praxisnah}

\section{Infektiologie-Update-Seminar}

Mit dem 9. Infektiologie-Update-Seminar erhalten Teilnehmer auch in diesem Jahr die Möglichkeit, sich in nur zwei Fortbildungstagen einen umfassenden Überblick über alle wichtigen Forschungsergebnisse des vergangenen

Jahres zu verschaffen. Das Infektio Update findet am 7. und 8. Mai 2021 in Mainz statt und überzeugt durch sein gewohntes Konzept: Aktuell, kompakt, praxisnah und neutral präsentieren und kommentieren hochkarätige Top-Experten in 14

\section{Programm}

\begin{tabular}{l}
$\begin{array}{l}\text { Sepsis } \\
\text { Stefan Kluge } \\
\text { Impfungen } \\
\text { Ulrich Seybold }\end{array}$ \\
$\begin{array}{l}\text { Gastrointestinale Infektionen } \\
\text { Thomas Weinke }\end{array}$ \\
ZNS-Infektionen \\
Matthias Klein \\
Influenza \\
Mathias Pletz \\
Haut- und Weichteilinfektionen \\
Christian Eckmann \\
\hline $\begin{array}{l}\text { Pneumonie } \\
\text { Jessica Rademacher }\end{array}$ \\
Covid-19 \\
Tobias Welte \\
Prävention nosokomialer Infektionen \\
Petra Gastmeier
\end{tabular}

Sexuell übertragbare Infektionen

Christoph D. Spinner

Hot Topic: Tropenmedizin \& parasitäre Erkrankungen Camilla Rothe

Hot Topic: Tuberkulose

Christoph Lange

Antibiotika und Antibiotic Stewardship

Rika Draenert

Pilzinfektionen

Oliver A. Cornely
Teilseminaren die relevantesten Studien der letzten zwölf Monate im Fachbereich Infektiologie.

Es erwartet Sie ein abwechslungsreiches Programm, das neben den wichtigsten Kernthemen in diesem Jahr um die Hot Topics "Tropenmedizin \& parasitäre Erkrankungen" und »Tuberkulose« ergänzt wurde.

Diskussionsrunden und Speakers' Corners runden die Veranstaltung ab und bieten wertvolle Gelegenheiten für den Austausch mit den Experten und Kollegen. Sie haben im Livestream die Gelegenheit, Ihre Fragen direkt über den interaktiven Livechat an die Referenten zu richten.

Jeder Teilnehmer erhält das Handbuch UPDATE INFEKTIOLOGIE 2021 mit ausführlichen Manuskripten aller Referenten - als Printausgabe oder im praktischen eBook-Format. Nach der Veranstaltung stehen sämtliche Vorträge als eCharts zum Download für die Teilnehmer zur Verfügung. Weiterhin können Teilnehmer der Veranstaltung sämtliche Vorträge kostenfrei auf unserer Videoplattform »streamedup!« noch einmal ansehen und anhören.

Unternehmen aus der Industrie unterstützen das Seminar, ohne dabei Einfluss auf die Inhalte zu nehmen. Die Veranstaltung, die erneut als paralleler Livestream stattfinden wird, steht unter der Schirmherrschaft der DGIM.

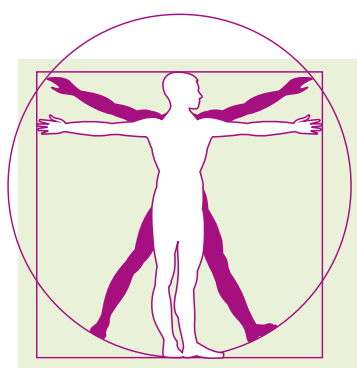

9. Infektiologie-UpdateSeminar

7. und 8. Mai 2021, Mainz

7. und 8. Mai 2021, Livestream

Wissenschaftliche Leitung Gerd Fätkenheuer, Köln Petra Gastmeier, Berlin Winfried V. Kern, Freiburg Mathias Pletz, Jena

Alle Veranstaltungen parallel als Livestream

UPDATE-Vorschau

Angio Update

12.-13.03.2021, Berlin

angio-update.com

Rheuma Update

12.-13.03.2021, Mainz

rheuma-update.com

Gastro Update

12.-13.03.2021, Berlin 19.-20.03.2021, Mainz gastro-update.com

\section{Allergo Update}

19.-20.03.2021, Mannheim allergo-update.com

Hämostase Update

23.-24.04.2021, Mainz haemostase-update.com

\& SCHIRMHERRSCHAFT Deutsche Gesellschaft für Innere Medizin

Überzeugt vom Update-Konzept übernimmt die DGIM seit 2007 die Schirmherrschaft für alle Update-Seminare der Inneren Medizin.

Sämtliche Informationen rund um die Veranstaltung finden Sie unter: infektio-update.com. 


\section{Veranstaltungen unter der Schirmherrschaft der DGIM}

\begin{tabular}{|c|c|c|}
\hline \multicolumn{3}{|c|}{ GI-Oncology 2021 - 17. Interdisziplinäres Update } \\
\hline $\begin{array}{l}\text { Ort der } \\
\text { Veranstaltung }\end{array}$ & Wiesbaden & $\begin{array}{l}\text { SCHIRMHERRSCHAFT } \\
\text { Deutsche Gesellschaft } \\
\text { für Innere Medizin } \\
\text { Ermäßigung für DGIM-Mitglieder }\end{array}$ \\
\hline Termin & \multicolumn{2}{|l|}{12.06 .2021} \\
\hline Veranstalter & \multicolumn{2}{|c|}{ MCI Deutschland GmbH } \\
\hline $\begin{array}{l}\text { Wissenschaftliche } \\
\text { Organisation }\end{array}$ & \multicolumn{2}{|c|}{ Prof. Dr. med. U. Graeven } \\
\hline Hauptthemen & \multicolumn{2}{|c|}{ Interdisziplinäres Update } \\
\hline $\begin{array}{l}\text { Info und } \\
\text { Anmeldung }\end{array}$ & \multicolumn{2}{|c|}{$\begin{array}{l}\text { MCI Deutschland GmbH } \\
\text { Telefon: +49 } 89549096-81 \\
\text { www.gi-oncology.de }\end{array}$} \\
\hline \multicolumn{3}{|c|}{ 2. Prevention Update } \\
\hline $\begin{array}{l}\text { Ort der } \\
\text { Veranstaltung }\end{array}$ & Kiedrich & $\begin{array}{l}\text { SCHIRMHERRSCHAFT } \\
\text { Deutsche Gesellschaft } \\
\text { für Innere Medizin } \\
\text { Ermäßigung für DGIM-Mitglieder }\end{array}$ \\
\hline Termin & \multicolumn{2}{|c|}{ 17.09.-18.09.2021 } \\
\hline Veranstalter & \multicolumn{2}{|c|}{$\begin{array}{l}\text { Deutsche Akademie für Präventivmedizin, } \\
\text { Rüdesheim } \\
\text { www.akaprev.de }\end{array}$} \\
\hline $\begin{array}{l}\text { Wissenschaftliche } \\
\text { Organisation }\end{array}$ & \multicolumn{2}{|c|}{ Dr. med. Johannes Scholl } \\
\hline Hauptthemen & \multicolumn{2}{|c|}{ Prävention - Innere Medizin } \\
\hline $\begin{array}{l}\text { Info und } \\
\text { Anmeldung }\end{array}$ & \multicolumn{2}{|l|}{ akaprev.de } \\
\hline
\end{tabular}

\begin{tabular}{|c|c|c|}
\hline $\begin{array}{l}\text { 58. Kongress de } \\
\text { Innere Medizin ( }\end{array}$ & $\begin{array}{l}\text { dwestde } \\
\text { (IM) }\end{array}$ & hen Gesellschaft für \\
\hline $\begin{array}{l}\text { Ort der } \\
\text { Veranstaltung }\end{array}$ & Heidelberg & $\begin{array}{l}\text { SCHIRMHERRSCHAFT } \\
\text { Deutsche Gesellschaft } \\
\text { für Innere Medizin }\end{array}$ \\
\hline & & Ermäßigung für DGIM-Mitglieder \\
\hline Termin & 08.10.-09.1C & 2021 \\
\hline Veranstalter & $\begin{array}{l}\text { Südwestdeu } \\
\text { zin e.V. (SWC }\end{array}$ & $\begin{array}{l}\text { che Gesellschaft für Innere Medi- } \\
\text { M) }\end{array}$ \\
\hline $\begin{array}{l}\text { Wissenschaftliche } \\
\text { Organisation }\end{array}$ & $\begin{array}{l}\text { Prof. Dr. mec } \\
\text { Univ.-Prof. D }\end{array}$ & $\begin{array}{l}\text { Martin Zeier } \\
\text { med. Dirk Jäger }\end{array}$ \\
\hline Hauptthemen & Innere Medi & \\
\hline $\begin{array}{l}\text { Info und } \\
\text { Anmeldung }\end{array}$ & $\begin{array}{l}\text { www.swgim } \\
\text { swgim } 2020\end{array}$ & $\begin{array}{l}\text { le/kongress } \\
\text { aey-congresse.de }\end{array}$ \\
\hline $\begin{array}{l}\text { 195. Tagung der } \\
\text { Innere Medizin ( }\end{array}$ & $\begin{array}{l}\text { leinisch-Wc } \\
\text { (GIM) }\end{array}$ & tfälischen Gesellschaft für \\
\hline $\begin{array}{l}\text { Ort der } \\
\text { Veranstaltung }\end{array}$ & Solingen & $\begin{array}{l}\text { SCHIRMHERRSCHAFT } \\
\text { Deutsche Gesellschaft } \\
\text { für Innere Medizin }\end{array}$ \\
\hline & & Ermäßigung für DGIM-Mitglieder \\
\hline Termin & 19.11.-20.1 & 2021 \\
\hline Veranstalter & $\begin{array}{l}\text { Rheinisch-W } \\
\text { Medizin e.V. }\end{array}$ & stfälische Gesellschaft für Innere \\
\hline $\begin{array}{l}\text { Wissenschaftliche } \\
\text { Organisation }\end{array}$ & Prof. Dr. mec & Hans Martin Hoffmeister \\
\hline Hauptthemen & $\begin{array}{l}\text { Innere Medi } \\
\text { heitlichem A }\end{array}$ & $\begin{array}{l}\text { n- Hohe Spezialisierung bei ganz- } \\
\text { satz }\end{array}$ \\
\hline $\begin{array}{l}\text { Info und } \\
\text { Anmeldung }\end{array}$ & www.rwgim & \\
\hline
\end{tabular}

Weitere Termine finden Sie unter https://www.dgim.de/fortbildung/veranstaltungskalender/ 


\section{Bad Nauheim}

19.04.-24.04.2021

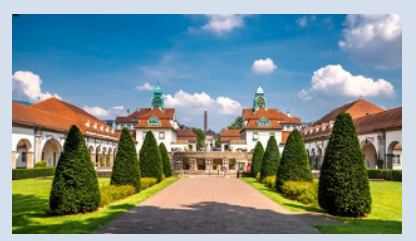

(c) pure-life-pictures

\section{Veranstaltungsort}

Fortbildungszentrum der Landesärztekammer Hessen Carl-Oelemann-Weg 5 61231 Bad Nauheim

\section{Gebühr}

Nichtmitglieder: 630,00 Euro DGIM-Mitglieder: 567,00 Euro Buchung einzelner Tage möglich

\section{Dresden}

06.09.-10.09.2021

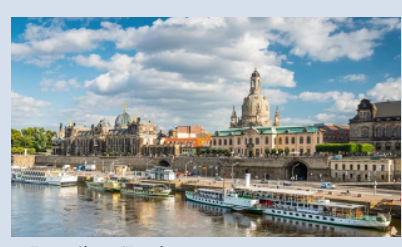

(c) Timo Christ/Fotolia

\section{Veranstaltungsort}

Akademie für med. Fort- und Weiterbildung der LÄK Schleswig-

Holstein

Esmarchstr. 4

23795 Bad Segeberg

\section{Gebühr}

Nichtmitglieder: 610,00 Euro DGIM-Mitglieder: 560,00 Euro

\section{Bad Segeberg \\ 06.09.-11.09.2021}

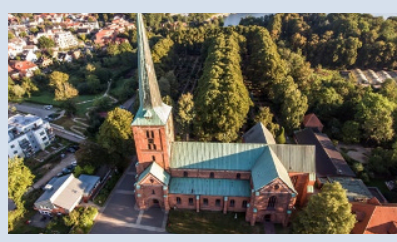

(o) Thomas Reimer/Fotolia

\section{Veranstaltungsort}

Akademie für med. Fort- und Weiterbildung der LÄK SchleswigHolstein

Esmarchstr. 4

23795 Bad Segeberg

\section{Gebühr}

Nichtmitglieder: 610,00 Euro DGIM-Mitglieder: 560,00 Euro
SCHIRMHERRSCHAFT
Deutsche Gesellschaft Deutsche Gesellsch
für Innere Medizin

\section{Veranstalter}

Akademie für Ärztliche Fort- und Weiterbildung der LÄK Hessen

\section{Leitung}

Prof. Dr. med. W. Fassbinder, Fulda

Anmeldung/Organisation

Akademie für Ärztliche Fort- und Weiterbildung

der LÄK Hessen

Adiela Candelo-Römer

Carl-Oelemann-Weg 5

61231 Bad Nauheim

Telefon: 06032 782-227

Telefax: 069 97672-67227

adiela.candelo-roemer@laekh.de www.akademie-laekh.de

SCCHIRMHERRSCHAFT Deutsche Gesellsch
für Innere Medizin

\section{Veranstalter}

Akademie für med. Fort- und Weiterbildung S-H, Bad Segeberg

\section{Leitung}

Prof. Dr. med. Heiner Mönig, Lübeck

\section{Anmeldung/Organisation}

Akademie für med. Fort- und Weiterbildung der LÄK Schleswig-

Holstein

Sylvia Kühne

Esmarchstr. 4

23795 Bad Segeberg

Telefon: 04551/803 76

Telefax: 04551/803 751

sylvia.kuehne@aeksh.de

\section{SCHIRMHERRSCHAFT Deutsche Gesellschat}

\section{Veranstalter}

Akademie für med. Fort- und Weiterbildung S-H, Bad Segeberg

\section{Leitung}

Prof. Dr. med. Heiner Mönig, Lübeck

Anmeldung/Organisation Akademie für med. Fort- und Weiterbildung der LÄK SchleswigHolstein

Sylvia Kühne

Esmarchstr. 4

23795 Bad Segeberg

Telefon: 04551/803 761

Telefax: 04551/803 751

sylvia.kuehne@aeksh.de

\section{Halle}

20.09.-24.09.2021

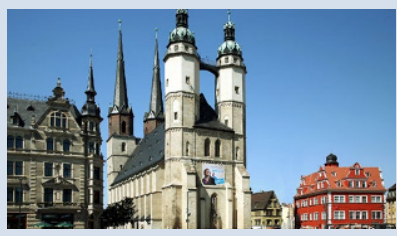

o Thomas Ziegler, Stadt Halle (Saale)

\section{Veranstaltungsort}

Universitätsklinikum Halle

Funktionsgebäude 6

Ernst-Grube-Straße 40

06120 Halle/Saale

\section{Gebühr}

Nichtmitglieder: 380,00 Euro DGIM-Mitglieder: 350,00 Euro

\section{München Rechts der Isar}

\subsection{9.-01.10.2021}

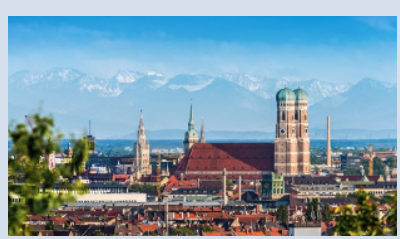

( ) euregiocontent

Veranstaltungsort

Klinikum rechts der Isar Hörsaal A

Ismaninger Straße 22

81675 München

Gebühr

Nichtmitglieder: 440,00 Euro DGIM-Mitglieder: 400,00 Euro

\section{Berlin}

25.10.-30.10.2021

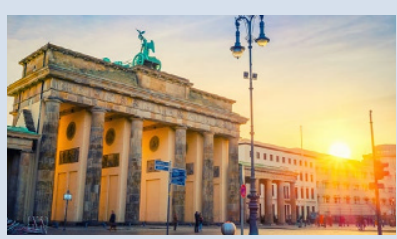

○ Sergey Borisov/Fotolia

\section{Veranstaltungsort}

Hotel Aquino

Tagungszentrum

Hannoversche Straße 5b

10115 Berlin-Mitte

\section{Gebühr}

Nichtmitglieder: 620,00 Euro DGIM-Mitglieder: 580,00 Euro

\section{SCHIRMHERRSCHAFT Deutsche Gesellschaft
für Innere Medizin}

\section{Veranstalter}

Universitätsklinikum Halle Department für Innere Medizin

\section{Leitung}

Prof. Dr. med. M. Binder Prof. Dr. med. M. Girndt Prof. Dr. med. P. Michl Prof. Dr. med. D. Sedding

\section{Anmeldung/Organisation} Universitätsklinikum Halle Stephanie Fritsche Department für Innere Medizin 06120 Halle/Saale

Telefon: 0345 557-4978 oder -3238 Telefax: 0345 557-904978 dim@uk-halle.de

\section{(3) SCHIRMHERRSCHAFT Deutsche Gesellsch
für Innere Medizin}

\section{Veranstalter}

Klinikum rechts der Isar, München

\section{Leitung}

Prof. Dr. med. R. M. Schmid Prof. Dr. med. F. Bassermann Prof. Dr. med. K.-L. Laugwitz

\section{Anmeldung/Organisation} PD Dr. med. Fabian Geisler Frau Mandy Söhner Klinikum rechts der Isar der TUM Klinik und Poliklinik für Innere Medizin II

Telefon: 089 4140-2252

Telefax: 089 4140-7287

mandy.soehner@mri.tum.de

SCHIRMHERRSCHAFT
Deutsche Gesellschaft
für Innere Medizin

\section{Veranstalter}

Medizinische Klinik I

Universitätsklinikum Lübeck Campus Lübeck

Ratzeburger Allee 160 23562 Lübeck

Leitung

Prof. Dr. G. Gerken Prof. Dr. G. Hintze Prof. Dr. H. Lehnert Prof. Dr. N. Suttorp

\section{Anmeldung/Organisation}

Frau D. Henze

Tel.: +49 1704904675

Fax: +4939162029414 intensivkurs-berlin@arcor.de www.persberg.de 